\section{Resultados clínicos de las enfermeras de práctica avanzada en heridas crónicas complejas en Andalucía}

\section{Clinical results of the nurses of advanced practice in complex chronic wounds in Andalucía}

Juan Francisco Jiménez García ${ }^{1, *}$

Gabriel Aguilera Manrique ${ }^{2}$

Francisco González Jiménez ${ }^{3}$

María Gutiérrez García ${ }^{4}$

Josefa Arboledas Bellón ${ }^{5}$

Francisco Pedro García Fernández ${ }^{6}$

1. Enfermero de Práctica Avanzada en Heridas Crónicas Complejas. Distrito Sanitario Poniente de Almería. Almería. España.

2. Profesor titular del Departamento de Enfermería. Facultad de Ciencias de la Salud. Universidad de Almería. Almería. España.

3. Enfermero de Práctica Avanzada en Heridas Crónicas Complejas. Distrito Sanitario Metropolitano de Granada. Granada. España.

4. Enfermera de Práctica Avanzada en Heridas Crónicas Complejas. Área de Gestión Sanitaria Serranía de Málaga. Málaga. España.

5. Enfermera de Práctica Avanzada en Heridas Crónicas Complejas. Distrito Sanitario Jaén Nordeste. Jaén. España.

6. Profesor del Departamento de Enfermería. Vicedecano de la Facultad de Ciencias de la Salud. Universidad de Jaén. Jaén. España.

*Autor para correspondencia.

Correo electrónico: juanfrajime@gmail.com (J.F. Jiménez García).

Recibido el 30 de abril de 2019; aceptado el 23 de mayo de 2019

\section{RESUMEN}

Objetivo: Describir y analizar el rol clínico de la enfermera de práctica avanzada en heridas crónicas complejas (EPA-HCC) en Andalucía, en cuanto al seguimiento de pacientes con heridas crónicas. Métodos: Estudio descriptivo transversal en los distritos Poniente de Almería, del A.G.C. Jaén Norte-Nordeste, A.G.S. Serranía de Málaga y Distrito Metropolitano de Granada entre junio del 2015 y junio del 2018, midiendo variables descriptivas como: diagnóstico etiológico de las heridas crónicas, tiempo de evolución antes de ser remitidos a EPAHCC, tasas de cicatrización, mejoría, empeoramiento, total de heridas crónicas, tasas de derivación desde EPA-HCC a hospitales. Resultados: Se realizó seguimiento de un total de 767 personas con heridas crónicas, donde el $70 \%$ de todas ellas fueron úlceras por presión, úlceras venosas

y lesiones de pie diabético, con una media de evolución de todas las lesiones de 21 meses y cuya derivación por parte de las EPA-HCC hacia los especialistas hospitalarios fue del 13,82\%, siendo más del 86,18\% ( $\mathrm{n}=661$ ) de los pacientes gestionados de forma eficaz por las EPA-HCC. La derivación directa de los pacientes a consulta de especialistas médicos desde la implantación de la figura fue tan solo del 5\%, con el importante ahorro en coste que esto supuso; además, las EPA-HCC atendieron a casi una de cada 4 heridas crónicas, datos estos que solo hicieron referencia a la atención directa, no de consultoría. Conclusiones: El rol clínico y coordinación que desempeñaron las EPA-HCC en los pacientes con heridas crónicas es efectiva en la mejora de los cuidados.

PALABRAS CLAVE: Enfermera de práctica avanzada, resultados clínicos, heridas crónicas, Andalucía.

\section{ABSTRACT}

Objective: To describe and to analyze the clinical role performed by the advanced practice nurse in complex chronic wounds (APN-CCW) in Andalusia, regarding the follow-up of patients with chronic wounds. Methods: Cross-sectional descriptive study in the Poniente of Almería Districts, of the H.M.A. Jaén NorthNortheast, H.M.A. Serranía de Málaga and the Metropolitan District of Granada between June 2015 and June 2018, measuring descriptive variables such as: etiological diagnosis of chronic wounds, evolution time before referring to $\mathrm{APN}-\mathrm{CCW}$, healing, improvement, worsening and deaths rates, total of chronic wounds, referral rates from APN-CCW to hospitals. Results: A total of 767 people with chronic wounds were followed up, and $70 \%$ of all of them were pressure ulcers, venous ulcers and diabetic feet, with an average of 21 months- evolution of all injuries and whose derivation from APN-CCW to hospital specialists was $13.82 \%$, with more than a $86.18 \%(n=661)$ of patients effectively managed by APN-CCW. The direct derivation of patients to the medical specialists office since the implementation of the figure was only $5 \%$, with the significant cost savings that this supposed, besides, the APN-CCW attended almost one in every 4 chronic wounds, no consulting. Conclusions: The clinical role and coordination of the APN-CCW in patients with chronic wounds is effective in improving care.

KEYWORDS: Advanced practice nurse, Clinical results, Chronic wounds, Andalusia. 


\section{У INTRODUCCIÓN}

En el marco del desarrollo de nuevas competencias y nuevos roles profesionales, se ha definido en el sistema sanitario de Andalucía como nuevo perfil específico el de enfermera de práctica avanzada en heridas crónicas complejas (EPA-HCC), que en su práctica asistencial integra cuatro roles: clínico experto, consultor, docente e investigador ${ }^{1}$. Las enfermeras que desarrollan este perfil son profesionales que ejercen un liderazgo clínico y constituyen un pilar clave para garantizar la calidad de la atención sanitaria, la seguridad clínica, la mejora en el acceso a los servicios, la mejora en la calidad de la atención sanitaria, optimizando el potencial de algunos grupos profesionales, la sostenibilidad del sistema y la satisfacción de los usuarios desde una práctica basada en la evidencia científica ${ }^{2,3}$. Desde el punto de vista clínico, la EPA-HCC proporciona conocimiento y habilidades utilizando razonamiento complejo, pensamiento crítico, reflexión como profesional experta en la práctica asistencial y ofrece consultas a las enfermeras y al equipo multidisciplinar para informar de las evaluaciones, de las tomas de decisiones y para diseñar los planes de atención a los pacientes críticamente enfermos o complejos ${ }^{4-8}$.

Los pacientes con heridas crónicas ${ }^{9}$, que son derivados a la EPAHCC, sufren con frecuencia un aumento del estrés psicosocial y de ansiedad, padecen un dolor continuo, con mala calidad de vida y que a veces ocasionan largos periodos de ingresos hospitalarios, ocasionándole al sistema sanitario elevados costes, por lo que las intervenciones clínicas efectivas y un abordaje eficaz de las EPAHCC se están convirtiendo cada día más en una necesidad dentro de los sistemas sanitarios ${ }^{10-16}$.

El propósito de este artículo es describir y analizar el rol clínico que realiza la EPA-HCC en Andalucía en cuanto al seguimiento de pacientes con heridas crónicas, antigüedad, evolución de las mismas y derivación a hospital para avanzar en las mejoras del paciente y de la organización.

\section{У METODOLOGÍA}

\section{Diseño}

Se planteó un estudio descriptivo transversal del rol clínico que desempeñaron la EPA-HCC en Andalucía, en los distritos Poniente de Almería, del A.G.C. Jaén Norte-Nordeste, A.G.S. Serranía de Málaga y Distrito Metropolitano de Granada (incorporado al estudio un año más tarde).

\section{Unidad de estudio}

Población: la población estudiada fueron todos los pacientes remitidos a las EPA-HCC por parte de las enfermeras y médicos de los distritos y áreas de gestión sanitaria anteriormente mencionados y que presentaban alguna lesión crónica.

Criterios de inclusión y exclusión: fueron incluidos todos los pacientes que cumplieron con los criterios de derivación previamente establecidos como fueron:

- Lesiones con evolución especialmente tórpida: entendidas como lesiones que ha tenido un manejo adecuado de la carga bacteriana de al menos 2 a 4 semanas; que presentaban alrededor de 6 semanas de puntuación de RESVECH mantenida; o con evolución superior a 12 semanas de la lesión sin signos claros de mejoría.
- Incapacidad para realizar el procedimiento de enfermería en su contexto asistencial: bien por falta de preparación real de los profesionales o el centro y no considerarse otras alternativas como adecuadas.

- Dudas sobre el criterio y pauta a seguir: tras la identificación adecuada del origen de la lesión y agotar todas las opciones terapéuticas más habituales de la misma.

- Otra situación que justifique la consulta de EPA-HCC.

Como criterios de exclusión se consideró:

- La imposibilidad de seguimiento del paciente por cualquier traslado a otro distrito de salud.

Tipo de muestreo: muestreo accidental o de conveniencias entre los pacientes que fueron derivados por parte de las enfermeras e incluidos en la recogida de datos por parte de las EPA-HCC, durante el periodo comprendido entre junio del 2015 y junio del 2018.

Tamaño de la muestra: se trató de una muestra conceptual por los criterios de inclusión antes propuestos; por tanto, se incluyeron todos los pacientes por los tipos de heridas crónicas durante el periodo de análisis.

\section{Variables del estudio analizadas}

- Diagnóstico etiológico de las heridas crónicas.

- Tiempo de evolución de las heridas antes de ser remitido a EPA-HCC.

- Tasas de cicatrización, tasas de mejoría, tasas de empeoramiento y de muertes.

- Total, de heridas crónicas por distritos.

- Tasas de derivación desde EPA-HCC a hospitales.

\section{Métodos e instrumentos para la recogida de datos}

Fue realizada por las EPA-HCC de los distritos anteriormente mencionados, a través de la herramienta de recogida de datos ad hoc de elaboración propia para tal fin. Además, se cumplimentaron los datos de la propia historia clínica de los pacientes y de las diferentes herramientas de heridas instaladas en DIRAYA, para el cálculo y análisis de los datos durante el periodo de 36 meses.

Los miembros que conformaron las EPA-HCC en Andalucía en los distritos anteriormente mencionados, junto con su coordinador, realizaron un análisis previo de las variables a analizar y qué resultados sería adecuado representar en función del tipo de heridas y de las características de estas. Una vez se desarrolló y consensuó el documento ad hoc, para la recogida de datos se creó el documento (en formato papel e informático) de recogida de datos y se procedió a la recolección de datos hasta alcanzar la recogida total.

\section{Análisis de los datos}

Se realizó un análisis descriptivo secuencial calculando las medidas de frecuencia y porcentaje para las variables cualitativas, y medidas de tendencia central (media y mediana) y dispersión (desviación estándar y rango) para las variables cuantitativas. Para su análisis se utilizó el programa Statistical Package for the Social Sciences en su versión 21.0.

\section{Aspectos éticos}

El presente estudio fue aprobado por el Comité de Ética de la Investigación de Centro Almería, como comité principal.

Este artículo forma parte de un proyecto más amplio en el que se pretendió medir a nivel global, y en determinados aspectos, la labor clínica de las EPA-HCC en Andalucía. 


\section{Y RESULTADOS}

A lo largo de los tres primeros años desde el inicio de la puesta en marcha de la EPA-HCC en Andalucía, en junio del 2015, se realizó la valoración, evaluación y seguimiento de un total de 767 personas con heridas crónicas, distribuidas en los distritos y áreas de gestión sanitaria como se muestra en la figura 1.

De los cuatro distritos de Andalucía donde se efectuó el estudio con las EPA-HCC se quiso señalar que los pacientes habían sido citados solo en los días establecidos para el desempeño del rol clínico, siendo 2 días a la semana en dos de los cuatro distritos y un día en semana en los otros restantes.

Como se puede observar en la figura 2, fueron las úlceras por presión, las úlceras venosas y el síndrome de pie diabético (úlceras neuropáticas, úlceras neuroisquémicas) las lesiones más prevalentes, con un $70 \%$ del total de las lesiones que fueron derivadas a las EPA-HCC por parte de los profesionales sanitarios, tanto desde atención primaria como de las residencias sociosanitarias.

\section{Seguimiento de lesiones (junio 2015-2018)}

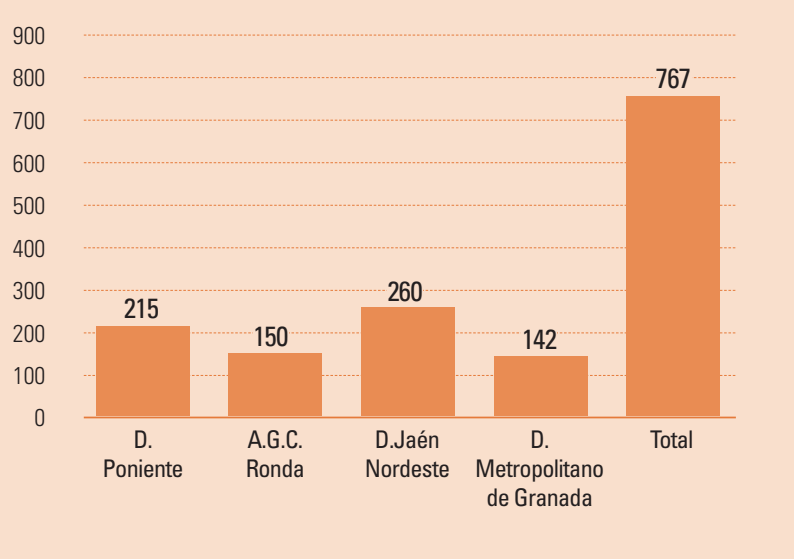

Figura 1. Seguimiento de personas con heridas crónicas.
En la misma figura 2 se incluyó en el tipo de lesiones el grupo "otras", que hace referencia a lesiones también crónicas menos prevalentes, con poca frecuentación pero no menos importantes, donde a los pacientes que las sufren y las padecen les ocasionan mucha sintomatología adversa, limitaciones funcionales, físicas, psicológicas, mala calidad de vida y largos periodos de convalecencia como fueron: epidermólisis bullosa, vasculitis, picaduras de araña, lesiones combinadas, autoinmunes, calcifilaxis.

Por otro lado, uno de los mayores problemas a los que se enfrentaron las EPA-HCC fueron los pacientes con lesiones crónicas, con medias de 21 meses con evolución muy tórpida y con un rango de amplitud en la variación de meses de evolución que iban desde un mes hasta llegar a alcanzar los 96, 372, 480 y 840 meses en los diferentes distritos como se puede observar en la figura 3 .

Dentro de la competencia clínica se analizaron las derivaciones de pacientes con heridas crónicas por parte de las EPA-HCC a las consultas de especialistas médicos hospitalarios durante el mismo periodo, y se pudo observar que ocasionó la derivación del $13,82 \%$ de los pacientes de un total de 767, siendo más del 86,18\% $(\mathrm{n}=661)$ de los pacientes gestionados de forma eficaz por las EPA-HCC.

Se pudo observar que la disminución de los pacientes a consulta de especialistas médicos desde la implantación de la figura fue de un 5\%, con el importante ahorro en coste que ello suposo y, por otro lado, las EPA-HCC hacían seguimiento de casi 1 de cada 4 heridas crónicas. Estos datos hacían referencia a la atención directa, porque el número de consultas telefónicas o por otras vías telemáticas fue mucho mayor, es decir, estos datos fueron de atención directa, no de consultoría.

Del total de 106 pacientes derivados a especialistas hospitalarios por circunstancias extremas y de urgencia, el 36,79\% fue derivado a las unidades de pie diabético, un 32,07\% a cirugía, un 9,43\% a cirugía vascular, un $7,54 \%$ a urgencias, un $6,60 \%$ a rehabilitación y un 1,88\% a nefrología; su derivación estaba indicada y fue efectiva en el 100\% de los casos (tabla 1).

A pesar de existir pacientes con heridas crónicas complejas de larga evolución, con mucha patología de base y enfermedades concomitantes que no favorecen el proceso normal de cicatrización, se consiguieron en los diferentes distritos y por las diferentes EPAHCC altas tasas de cicatrización y mejoría (RESVECH disminuido) que rondaron el $82,5 \%$ de los casos, como queda reflejado en la figura 4.

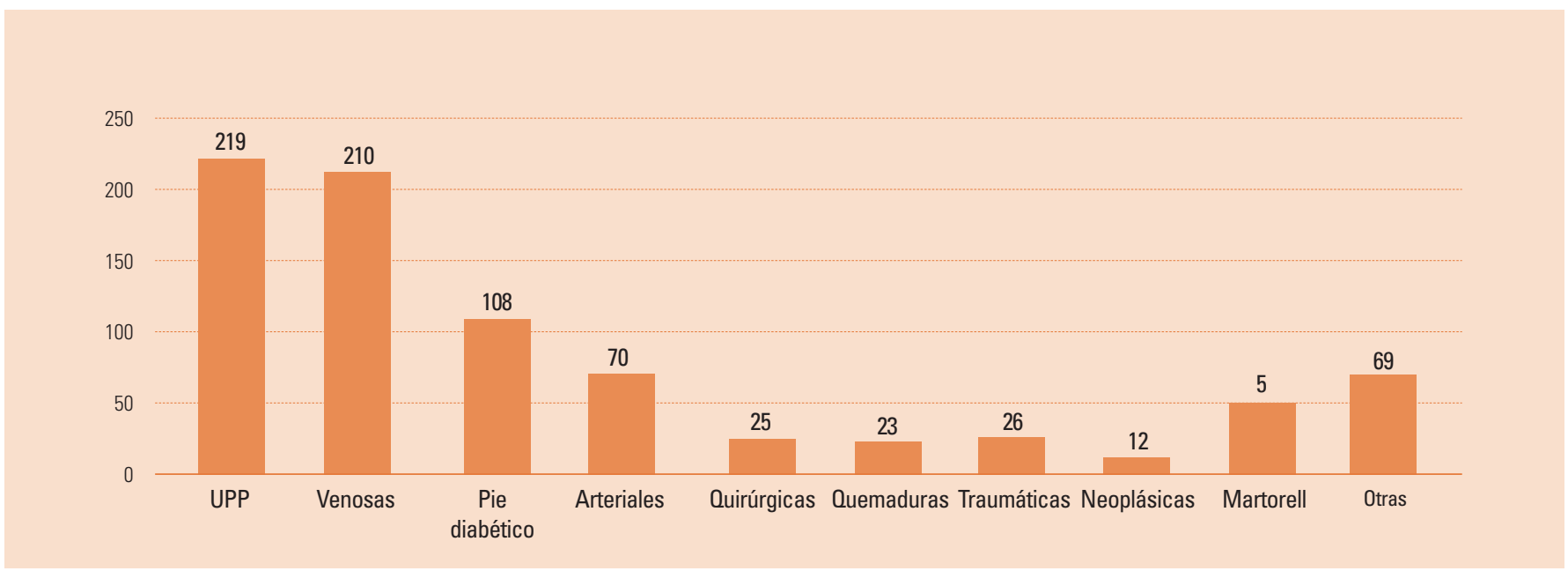

Figura 2. Lesiones crónicas más prevalentes. 


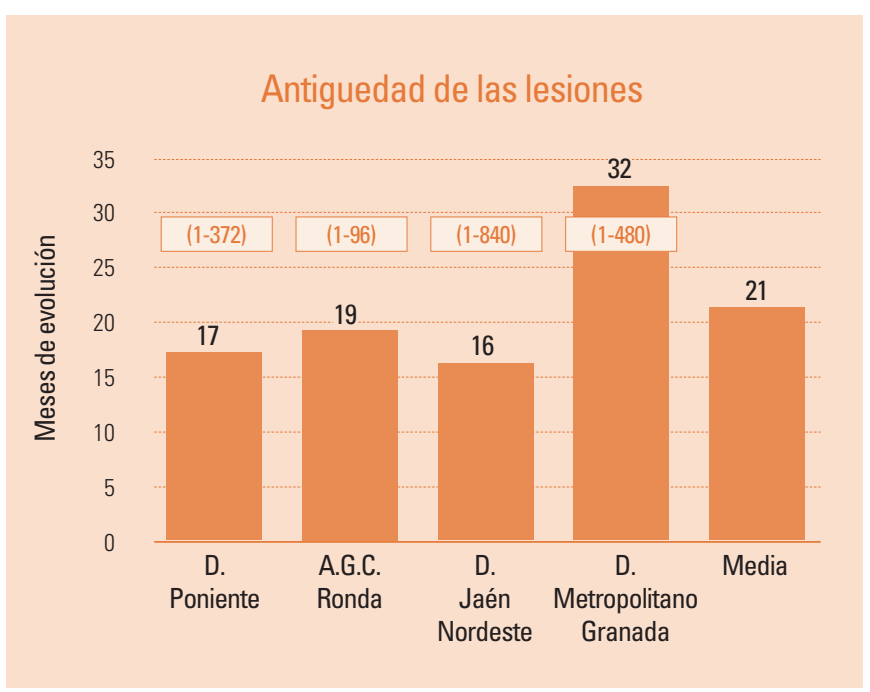

Figura 3. Antigüedad en meses de las lesiones.
Tabla 1. Derivación de EPA-HCC a especialistas hospitalarios

\begin{tabular}{|l|l|l|l|l|l|}
\hline & $\begin{array}{c}\text { D. } \\
\text { Poniente }\end{array}$ & $\begin{array}{c}\text { A.G.C. } \\
\text { Ronda }\end{array}$ & $\begin{array}{c}\text { D. Jaén } \\
\text { Nordeste }\end{array}$ & $\begin{array}{c}\text { Metropo- } \\
\text { litano de } \\
\text { Granada }\end{array}$ & Total \\
\hline $\begin{array}{l}\text { U. pie } \\
\text { diabético }\end{array}$ & 2 & 0 & 0 & 37 & 39 \\
\hline Cirugía & 13 & 14 & 7 & 0 & 34 \\
\hline C. vascular & 0 & 0 & 8 & 2 & $\mathbf{1 0}$ \\
\hline Urgencias & 0 & 0 & 0 & 8 & $\mathbf{8}$ \\
\hline Dermatología & 4 & 2 & 0 & 1 & $\mathbf{7}$ \\
\hline Rehabilitación & 2 & 0 & 1 & 3 & $\mathbf{6}$ \\
\hline Nefrología & 0 & 2 & 0 & 0 & $\mathbf{2}$ \\
\hline
\end{tabular}

\section{Evolución de las lesiones}

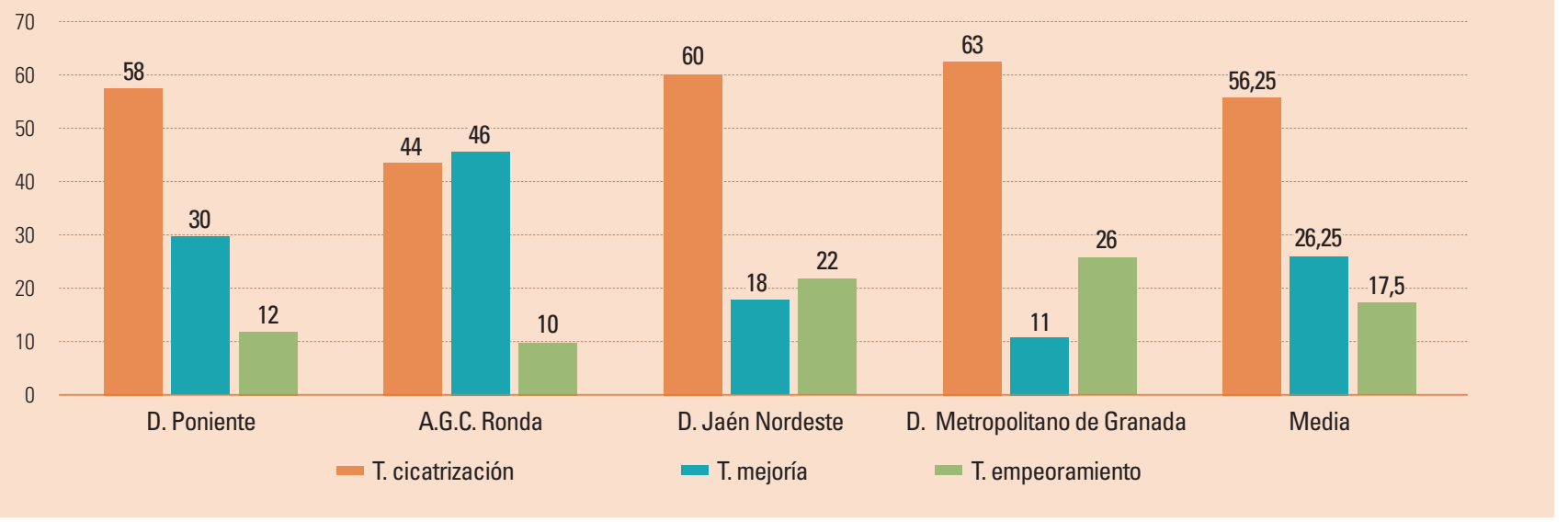

Figura 4. Porcentajes y evolución de las lesiones.

\section{У DISCUSIÓN}

Queremos destacar que los datos presentados en este artículo ofrecen una visión de la situación que existe en los distritos sanitarios andaluces con respecto al análisis y seguimiento que realizan las EPA-HCC a los pacientes que presentan heridas crónicas complejas.

Nos pareció importante reseńar que las cuatro EPA-HCC en los distritos indicados dan cobertura a una población que sobrepasa la cifra de 1.360.000 habitantes, lo que supone el 16,18\% de la población andaluza; si estamos confirmando que se ha realizado el seguimiento a un total de 767 personas con lesiones crónicas, podríamos estimar y estar hablando, al extrapolar los datos, que en Andalucía podría haber aproximadamente unas 4.740 personas con heridas crónicas, muchísimas de ellas complejas y que no están siendo atendidas por EPA-HCC, lo que podría conllevar el aumento en los recursos de personal, de materiales, de ingresos hospitalarios, de gasto público y el aumento en la mala calidad de vida de los pacientes.
También nos parece importante resaltar que de todos los pacientes que fueron derivados a EPA-HCC, más del 70\% de ellos presentaban lesiones crónicas como son las úlceras por presión, las úlceras venosas y el pie diabético, lo que supone actualmente un grave problema de salud pública en los países desarrollados, al igual que en nuestro país y en nuestra comunidad. Los beneficios clínicos y económicos tanto para los pacientes como para el sistema sanitario público podrían derivarse de estrategias que se centran en la prevención de heridas, el diagnóstico preciso, en mejorar las tasas de cicatrización, en reducir las tasas de ingresos hospitalarios, en disminuir la infección, las amputación y muertes como así manifiestan dichos autores ${ }^{17-21}$.

Además, nos pareció importante incluir el tiempo de evolución en cuanto a la antigüedad de las lesiones que han sido vistas en los diferentes distritos sanitarios, que ronda de media casi 2 años. Esto pone de manifiesto que las heridas crónicas no solamente ocasionan grandes costes económicos a los países y a sus sistemas sanitarios, sino que provocan dolor crónico continuo y mala calidad de vida de las personas que las padecen, lo que coincide con los estudios de otros 
investigadores ${ }^{14,22-26}$ y hace más evidente la labor clínica de la EPA$\mathrm{HCC}$ en estos casos.

Las derivaciones de pacientes con heridas crónicas desde las EPAHCC a especialistas hospitalarios han sido mínimas a lo largo de estos 3 ańos de análisis, con tan solo uno de cada diez, lo que conlleva que muy mayoritariamente son gestionadas de forma eficaz por las EPAHCC en Andalucía, y esto se traduce en un importante ahorro en coste, un impacto positivo de las intervenciones y la efectividad desde la atención primaria. Esta posición es compartida por otros autores de países anglosajones donde ya tienen consolidada desde hace muchos años esta figura $^{6,7,15,27-30}$.

Sin embargo, y a pesar de obtener resultados más que aceptables en cuanto a tasas de cicatrización y mejoría tras el seguimiento y actuación por parte de las EPA-HCC, este estudio presenta algunas limitaciones que no podemos dejar de señalar, como son las tasas de empeoramiento del 16\%, una cifra, aunque baja de forma global, demasiado alta para la expectativa de las EPA en HCC.

Por otro lado, también queremos señalar el porcentaje de pacientes que han muerto durante el seguimiento por las EPA-HCC (12\%), lo cual nos debe hacer reflexionar sobre si las derivaciones y la aceptación de estas fueron adecuadas, o por el contrario primó más ver la lesión que la situación global del paciente y unos objetivos terapéuticos reales.

\section{$\searrow$ CONCLUSIONES}

- Conocer el tipo de lesiones y su antigüedad permite establecer estrategias de mejora en cuanto a la formación a desarrollar y a las medidas preventivas a adoptar.

- La mayoría de las heridas atendidas son lesiones por presión o lesiones de la extremidad inferior.

- Las lesiones son muy tórpidas, con una media que ronda los 2 años de evolución clínica, con los evidentes costes para el paciente y el sistema.

- La tasa de cicatrización o mejoría de estas lesiones ha sido del $82,5 \%$ de media en los cuatro distritos sanitarios.

- La tasa de derivaciones al hospital ha sido del 13\%, lo que sumado al punto anterior supone un importante ahorro en el tratamiento de estos pacientes.

- La coordinación es clave en la mejora de la continuidad de cuidados, desempeñando en este caso la EPA-HCC un rol clínico efectivo.

\section{Agradecimientos}

A todas las enfermeras que participaron en este proyecto. A todas las enfermeras que forman parte de la Estrategia de Cuidados de Andalucía. A los pacientes que cada día hacen que nuestro trabajo sea mejor.

\section{Conflicto de intereses}

Los autores no declaran ningún conflicto de interés.

\section{Y BIBLIOGRAFÍA}

1. Junta de Andalucía. Consejería de Salud. Enfermera de Práctica Avanzada en la atención de personas con Heridas Crónicas Complejas (EPA-HCC). Sevilla: Servicio Andaluz de Salud; 2018.

2. Camacho-Bejarano R, Rodríguez-Gómez S, Padín-López S, Lima-Serrano M. Enfermería Clínica, pisando fuerte. Enferm Clin. 2015;25(1):1-2

3. Camacho Bejarano R, Rodríguez Gómez S, Gómez Salgado J, Lima Serrano M. Padín López S. Calidad, accesibilidad y sostenibilidad: Claves para la Enfermería de Práctica Avanzada en España. Enferm Clin. 2015; 25(5):221-2.

4. Chamblee TB, Urden LD, Ray MM, Moody R, Mayo AM. The Advanced Practice Clinical Nurse Specialist. Nurs Adm 0 2016:41(1):70-6.

5. Lamb A, Martin R, Bryant D, Latimer M. Describing the leadership capabilities of advanced practice nurses using a qualitative descriptive study. Nurs Open. 2018; 5(3):400-13

6 . Pearce C, Breen B. Advanced clinical practice and nurse-led clinics: a time to progress. Br J Nurs. 2018; 27(8):444-8.

7. Fagerström $L$. The impact of advanced practice nursing in healthcare recipe for developing countries. Ann Neurosci. 2012;19(1):1-2

8. O'Connell J, Gardner G, Coyer F. Beyond competencies: Using a capability framework in developing practice standards for advanced practice nursing. J Adv Nurs. 2014;70(12):2728-35.

9. García-Fernández FP, López-Casanova P, Segovia-Gómez T, Soldevilla- Agreda JJ, Verdú-Soriano J. Unidades Multidisciplinares de Heridas Crónicas: Clínicas de Heridas. Serie Documentos de Posicionamiento GNEAUPP $n^{0} 10$. Grupo Nacional para el Estudio y Asesoramiento de Úlceras por Presión y Heridas Crónicas. Logroño; 2012
10. González-Consuegra RV. Verdú J. Proceso de adaptación al casteIlano del Charing Cross Venous UIcer Questionnaire (CCVUO) para medir la calidad de vida relacionada con la salud en pacientes con úlceras venosas. Gerokomos. 2010;21(2):80-7.

11. Mclain N, Moore Z. Wound cleansing for treating venous leg ulcers. Cochrane Database Syst Rev. 20154:CD011675

12. Adderley UJ, Thompson C. A comparison of the management of venous leg ulceration by specialist and generalist community nurses: A judgement analysis. Int J Nurs Stud. 2016; 53:134-43.

13. Guy H. Now is the time for tissue viability. Br J Nurs. 2011;20(11):S30-S30.

14. Hemingway B, Storey C. Role of the clinical research nurse in tissue viability. Nurs Stand. 2013;27(24):62-8.

15. Palfreyman $\mathrm{S}$. The value of nurse-led tissue viability services. $\mathrm{Br}$ J Nurs. 2012:21(20):3

16. Milne J, Ousey K. Tissue viability $2010-2015$ : from good to great. Br J Community Nurs. 2010; Suppl:S18, S20, S22.

17. Guest JF, Fuller GW, Vowden P. Venous leg ulcer management in clinical practice in the UK: costs and outcomes. Int Wound $\mathrm{J}$. 2018;15(1):29-37

18. García-Fernández FP, Pancorbo-Hidalgo PL, López-Ortega J, LópezMedina IM. Recursos materiales para la prevención y el tratamiento de las úlceras por presión: análisis de la situación en Andalucía. Gerokomos. 2006;17(1):47-57.

19. Guest JF, Fuller GW, Vowden P. Diabetic foot ulcer management in clinical practice in the UK: costs and outcomes. Int Wound $\mathrm{J}$. 2018;15(1):43-52

20. Pancorbo PL, García FP, Torra JE, Verdú J, Soldevilla JJ. Epidemiología de las úlceras por presión en España en 2013 : $4^{\circ}$ Estudio Nacional de Prevalencia. Gerokomos. 2014; 25(4):162-70.
21. Demarré L, Van Lancker A, Van Hecke A, Verhaeghe S, Grypdonck M, Lemey J, et al. The cost of prevention and treatment of pressure ulcers: A systematic review. Int J Nurs Stud. 2015 52(11):1754-74

22. Tilley C, Lipson J, Ramos M. Palliative Wound Care for Malignant Fungating Wounds: Holistic Considerations at End-of-Life. Nurs Clin North Am. 2016;51(3):513-31.

23. Newbern S. Identifying Pain and Effects on Quality of Life from Chronic Wounds Secondary to Lower-Extremity Vascular Disease: An Integrative Review. Adv Ski Wound Care. 2018;31(3):102-8.

24. Howell RS, Gorenstein S, Gillette BM, DiGregorio J, Criscitelli T, Davitz MS, et al. A Framework to Assist Providers in the Management of Patients with Chronic, Nonhealing Wounds. Adv Skin Wound Care. 2018;31(11):491-501.

25. Frescos N. Assessment of pain in chronic wounds: A survey of Australian health care practitioners. Int Wound J. 2018;15(6):943-9.

26. Fauziyah $\mathrm{H}$, Gayatri D. Pain, stress, and sleep quality in chronic wound patients. Enferm Clin. 2018;28 Suppl 1:176-9.

27. Gray M. Context for WOC practice: Validating our assessments and interventions. J Wound Ostomy Continence Nurs. 2010;37(5):455-7.

28. Bliss DZ, Westra BL, Savik K, Hou Y. Effectiveness of Wound, Ostomy and Continence-Certified Nurses on Individual Patient Outcomes in Home Health Care. J Wound Ostomy Continence Nurs. 2013:40(2):135-42.

29. Gray M. Context for practice: The renaissance WOC nurse: Expert clinician, educator, clinical investigator, leader, and mentor. J Wound Ostomy Continence Nurs. 2014;41(1):11-2.

30. Cowan T. Management solutions for tissue viability: JWC conference report. J Wound Care. 2010;19(4):161, 163-6. 